\title{
Milliliter per 24 Hours
}

National Cancer Institute

\section{Source}

National Cancer Institute. Milliliter per 24 Hours. NCI Thesaurus. Code C67410.

A metric unit of volumetric flow rate defined as the rate at which one milliliter of matter crosses a given surface during the period of time equal to 24 hours. 